\title{
Percepção dos treinadores sobre as competências profissionais em função da sua formação e experiência
}

\author{
Coaches' perception of professional competence as a function of \\ education and experience
}

Ana Sofia Figueiredo Marques dos Santos Isabel Maria Ribeiro Mesquita

1 Universidade do Porto, Faculdade de Desporto. Porto, Portugal.

Recebido em 22/09/09 Revisado em 19/11/09 Aprovado em 23/03/10
Resumo - O objectivo deste estudo consistiu na análise da valorização percebida de competências profissionais em função da experiência profissional e da formação académica de treinadores. A amostra foi constituída por 343 treinadores portugueses, oriundos de diferentes modalidades. A recolha dos dados foi garantida pela aplicação de um questionário após validação de construção e de conteúdo. Como procedimentos estatísticos, recorreu-se à análise factorial exploratória e à análise inferencial através do teste one-way ANOVA. Da análise das competências percebidas como importantes, resultaram 5 factores: planeamento; liderança e formação de treinadores; planeamento e orientação das competições; aspectos pessoais; e orientação do treino. Apesar de todas terem sido consideradas, no mínimo, com importância razoável, as relacionadas com o contexto da competição destacaram-se como as mais valorizadas. A experiência profissional e a formação académica apresentaram-se como factores diferenciadores da valorização atribuída às competências. Os treinadores com mais experiência concederam mais importância às competências relacionadas com o planeamento, à orientação do treino, à liderança e à formação de treinadores em relação aos treinadores pouco experientes. Os treinadores com formação superior valorizaram mais as competências de planeamento, liderança e formação de treinadores, orientação do treino e competências pessoais do que os treinadores com o $2^{\circ}$ grau completo.

Palavras-chave: Treinadores; Competência profissional; Experiência; Formação académica.

Abstract - The aim of this study was to analyze coaches' perception of professional competence as a function of experience and academic education. The sample consisted of 343 Portuguese coaches from different sport disciplines. A validated questionnaire was used to collect demographic data and data regarding the perception of professional competence. Exploratory factorial analysis and comparative inferential analysis by one-way ANOVA were used as statistical procedures. The results highlighted the wide range of competences necessary for an efficient coach. The answers provided by the coaches revealed 5 factors: planning; leadership and coach education; competition planning and orientation; personal competence, and training orientation. Although all these factors were at least classified as reasonably important, factors related to competition were considered to be the most important. Comparative analysis showed that professional experience and academic education had a different impact on the perceived importance of competence. More experienced coaches perceived competences related to planning, training orientation, and leadership and coach education to be more important than less experienced coaches. Coaches with higher education considered competences related to planning, leadership and coach education, training orientation and personal competence to be more important than undergraduate coaches.

Key words: Coach; Professional competence; Professional experience; Academic education. 


\section{INTRODUÇÃO}

Dada a proeminência do papel social do desporto na sociedade actual, tem-se vindo a verificar um incremento na importância atribuída ao papel do treinador, aos seus conhecimentos e suas competências $^{1}$. Da investigação orientada para o estudo do comportamento dos treinadores, prevalecente nas décadas de 1970 e 1980, a agenda da investigação direccionou-se, gradativamente, para os aspectos cognitivos que sustentam as atitudes dos treinadores, examinando as crenças e conhecimentos destes ${ }^{2,3}$. Daí, tem-se o resultado que a intervenção do treinador no processo de treino e de competição é multifacetada e desenvolvida em múltiplos contextos de prática, requerendo, assim, o domínio de um largo e exigente leque de conhecimentos e competências. Nesta conformidade, as competências profissionais, face a exigências do contexto de prática, têm vindo a ser alvo da atenção da investigação ${ }^{4-8}$.

Não raramente, os conceitos de conhecimento e competência são referidos como sinónimos, sendo estudados de forma não sistemática e diferenciada. Apesar do conceito de conhecimento possuir diversas definições, a literatura é unânime em considerar que se trata da representação de um domínio de conceitos ou princípios teóricos, que são aprendidos, lembrados ou reproduzidos ${ }^{5,9}$. Porém, o conceito não inclui uma conotação de aplicação, sendo necessária uma mudança de paradigma na investigação, evoluindo das questões relativas àquilo que os treinadores devem saber para a habilidade de aplicar aquilo que sabem ${ }^{4}$, sendo crucial à competência o facto de ser específica relativamente às circunstâncias nas quais se desenrola ${ }^{10}$.

A literatura refere um alargado leque de competências de base, reportado aos contextos do treino, da competição e da organização $0^{4,11-13}$. Nomeadamente, os programas de formação de treinadores previstos pelo projecto europeu $\mathrm{AEHESIS}^{13}$ e pelo programa canadiano $\mathrm{BIS}^{4}$ indicam que o treinador deverá ser capaz de realizar tarefas como organizar, implementar, avaliar e ajustar um planeamento e orientar os atletas durante o treino e a competição ${ }^{4,13}$. Por outro lado, as tarefas desempenhadas pelos treinadores exigem que estes se assumam como líderes de programas de formação e de desenvolvimento desportivo, pelo que a liderança e a gestão adquirem um importante papel no desempenho do treinador ${ }^{4,7,13}$.

Finalmente, na base da intervenção do treinador, situam-se as competências pessoais, nomeadamente, a capacidade de comunicação, de reflexão e de responsabilidade pessoal ${ }^{13}$, que sustentam a sua interacção e relacionamento com todos os intervenientes no desporto ${ }^{14,15}$.

Assim, existe uma panóplia de competências a serem adquiridas pelos treinadores, pelo que se torna importante perceber aquelas que os treinadores mais valorizam na sua intervenção profissional. De facto, entre os factores concorrentes da competência profissional, a competência percebida é considerada como nuclear na competência efectiva ${ }^{5}$, sendo de grande importância o seu estudo para se intentar compreender a complexidade do processo de treino e, concomitantemente, indicar novas orientações para a formação de treinadores.

O estudo das concepções dos treinadores ganha então pertinência, nomeadamente, na informação que faculta ao desenvolvimento de programas de formação, através de um entendimento mais profundo do processo de treino e, ainda mais, quando associado a variáveis afetas às características dos treinadores, como seja a formação académica ${ }^{4}$ e a experiência profissiona ${ }^{13}$. Enquanto a experiência profissional tem sido indicada como promotora de aprendizagem e de evolução dos treinadores em direcção à expertise ${ }^{3,8,13-18}$, dado o suporte pedagógico e didáctico fornecido nos cursos de Educação Física e Desporto a formação académica tem mostrado ser qualificadora da intervenção profissional ${ }^{4,19}$.

Perante este enquadramento teórico, o presente estudo pretende responder a duas grandes questões de pesquisa: Quais são as competências profissionais que os treinadores mais valorizam para exercer a sua actividade? Será que a valorização destas competências varia em função da experiência profissional e da formação académica dos treinadores? O objectivo deste estudo consistiu em analisar a valorização percebida de competências profissionais, em função da experiência profissional e da formação académica de treinadores.

\section{PROCEDIMENTOS METODOLÓGICOS}

\section{Amostra}

Participaram 343 treinadores portugueses (289 do sexo masculino; 54 do sexo feminino), provenientes de 22 modalidades cuja idade variou entre os 16 e os 65 anos de idade $(32,37 \pm 9,84)$. Os participantes registaram a sua experiência profissional desde 1 ano a 25 anos de experiência $(5,94 \pm 6,47)$. A categorização da referida experiência baseou-se em dois pressupostos: considerar que 5 anos de experiência são o limite mínimo para o treinador possuir alguma experiência ${ }^{20} \mathrm{e}$ o facto dos 10 anos 
ser a referência apontada na literatura como indicador da posse de uma experiência substantiva, em busca da expertise, ou seja, da mestria profissional ${ }^{7}$. Assim, foram criadas as categorias: a) treinadores pouco experientes ( $\mathrm{n}=129 ; 38,6 \%)$, na qual foram incluídos os sujeitos com 1 a 5 anos de experiência; b) experientes ( $\mathrm{n}=53 ; 15,9 \%)$, com 5 a 9 anos de experiência; e c) muito experientes, ou seja, com mais de 10 anos ( $n=152 ; 45,5 \%)$.

Uma vez que a formação superior pode diferenciar os valores e competências dos treinadores, a formação não superior foi distinguida da superior, criando-se a categoria até $o 2^{\circ}$ grau completo $(n=142$; 42,1\%). Os cursos de Educação Física e Desporto, por abordarem especificamente matérias adstritas às Ciências do Desporto, podem promover conhecimentos e competências relativas ao papel do treinador $^{4,19}$, o que levou à formação de dois grupos: treinadores com formação superior em Educação Física (E.F.) ( $n=154 ; 45,7 \%)$ e treinadores com outras formações superiores $(\mathrm{n}=41 ; 12,2 \%)$.

\section{Procedimentos}

Na recolha dos dados, foi utilizado um questionário construído por meio da consulta da literatura da especialidade $e^{5,712,13}$. De seguida, o questionário foi objecto de validação de conteúdo, tendo sido sujeito a uma apreciação por parte de um painel de três peritos doutorados em Ciências do Desporto e especialistas na temática, os quais desenvolveram uma análise item a item, gerando algumas reformulações. Finalmente, o instrumento foi objecto de uma aplicação piloto, no sentido de se aferir acerca da inteligibilidade, nível de compreensão, clareza e objectividade das questões. Neste sentido, foi aplicado a 30 treinadores com intervenção em diferentes modalidades e escalões de prática e, ainda, com experiência profissional diferenciada. A versão final do questionário é composta por duas partes; a primeira foca as características do treinador e a segunda a valorização das competências pela caracterização de 37 itens por meio de uma escala Likert que varia entre sem importância (0) a muito grande importância (5).

Os questionários foram preenchidos por treinadores presentes em congressos e seminários referentes à formação de treinadores. Esclarecidos os objectivos do estudo, os investigadores asseguraram que a identidade dos voluntários se manteria anónima no estudo, sendo obtida uma declaração de consentimento livre e esclarecido.

As propriedades psicométricas do instrumento foram avaliadas por meio de análise factorial exploratória. Com o intuito de simplificar a interpre- tação dos dados, foi utilizado o método de rotação Varimax, que identificou os factores emergentes ${ }^{21}$. Para verificar a validade dos factores, apenas foram considerados aqueles com mais de 3 itens e com um valor mínimo de eigenvalue de 1.0. $O$ teste de Kaiser-Meyer-Olkin (KMO) verifica a adequação dos dados para a aplicação de uma análise desta natureza, enquanto o teste de esfericidade de Bartlett indica a força da relação entre as variáveis. Visto que o primeiro apontou para valores superiores a 0,5 $(\mathrm{KMO}=0,855)$ e o segundo resultou em diferenças significativas $(\mathrm{p}<0,01)$, verificaram-se os pressupostos para a aplicação de uma análise factorial. Os loading scores dos factores apresentaram-se acima do valor de referência (0.50) e os factores revelaram elevada consistência interna (alpha de Cronbach acima do valor de $0.70^{21}$.

Finalmente, recorreu-se à estatística descritiva para calcular frequências, médias e desvios padrões e ao teste paramétrico de análise de variância one-way ANOVA, estimando-se os Post-Hocs por meio do teste de Tukey, de modo a indagar possíveis diferenças nas percepções dos treinadores em função da sua experiência profissional e formação académica.

\section{RESULTADOS}

Da análise factorial emergiram cinco factores (Quadro 1) que explicaram $63,40 \%$ da variância total. Os treinadores valorizaram as competências profissionais referentes ao planeamento (factor 1), que apresentaram uma média de 3,86; à liderança e formação de treinadores (factor 2), com uma média de 3,96; ao planeamento e orientação da competição (factor 3) (média de 4,18); às características pessoais (factor 4) (média de 3,84); e, finalmente, à orientação do treino (factor 5) (média de 3,96). Estes valores apontam para o facto de os treinadores avaliarem, em média, as competências referidas pelos factores como possuindo entre importância razoável e grande importância.

A análise comparativa dos cinco factores em função da experiência profissional dos treinadores apontou três diferenças significativas na valorização das competências. Os treinadores muito experientes valorizaram significativamente mais o factor $1\left(\mathrm{~F}_{2,326}=3,496 ; p=.025\right)$ e o factor $5\left(\mathrm{~F}_{2,330}=6,984\right.$; $\mathrm{p}=.001)$ do que os treinadores pouco experientes. Os treinadores experientes valorizaram, significativamente, mais $\left(\mathrm{F}_{2,328}=4,474 ; \mathrm{p}=.016\right)$ o factor 2 do que os pouco experientes.

Na formação académica, foram identificadas cinco diferenças significativas. Os treinadores com formação superior em Educação Física valorizaram, sig- 
nificativamente, mais $\left(\mathrm{F}_{2,322}=12,762 ; \mathrm{p}=.000\right)$ o factor 5 do que os com o $2^{\circ}$ grau completo. Os treinadores com outras formações superiores classificaram como mais importante o factor $1\left(\mathrm{~F}_{2,329}=5,400 ; \mathrm{p}=.003\right)$, o factor 2 $\left(\mathrm{F}_{2,331}=2,993 ; \mathrm{p}=.040\right)$, o factor $4\left(\mathrm{~F}_{2,326}=4,831 ; \mathrm{p}=.008\right)$ e o factor $5\left(\mathrm{~F}_{2,333}=12,762 ; \mathrm{p}=.000\right)$ relativamente aos treinadores com o $2^{\circ}$ grau completo.

\section{DISCUSSÃO}

Os resultados confirmaram a valorização, por parte dos treinadores, da diversidade de competências para o exercício eficaz da actividade ${ }^{7}$, classificadas de importância razoável a grande importância na totalidade dos itens.

Particularmente, as competências relativas ao planeamento foram consideradas de grande importância, surgindo como o primeiro factor. Estes resultados vêm confirmar a tendência encontrada na literatura, na medida em que tanto a investigação na área ${ }^{7,22}$, como a derivada de programas de formação de treinadores recentes ${ }^{4,13}$, têm referido a capacidade de planear como uma tarefa crucial do treinador. Côté \& Sedgwick ${ }^{11}$ afirmam que a inves-

Quadro 1. Matrix factorial resultante da análise das componentes principais.

\begin{tabular}{|c|c|c|c|c|c|c|c|}
\hline & & & Factores / Itens & Loadings & Alpha & $\begin{array}{l}\text { Eigen } \\
\text { values }\end{array}$ & $\begin{array}{l}\text { \% Vari- } \\
\text { ância }\end{array}$ \\
\hline \multirow[t]{9}{*}{1} & \multirow[t]{9}{*}{$\begin{array}{l}\text { Competências de } \\
\text { Planeamento }\end{array}$} & 6 & $\begin{array}{l}\text { Organizar e implementar o planeamento } \\
\text { plurianual. }\end{array}$ & 0,856 & \multirow[t]{9}{*}{0,894} & \multirow[t]{9}{*}{7,282} & \multirow[t]{9}{*}{31,662} \\
\hline & & 3 & $\begin{array}{l}\text { Realizar plano de preparação plurianual, } \\
\text { considerando as necessidades do atleta/ } \\
\text { grupo. }\end{array}$ & 0,828 & & & \\
\hline & & 9 & Avaliar o plano de preparação plurianual. & 0,788 & & & \\
\hline & & 12 & Estabelecer plano plurianual na competição. & 0,775 & & & \\
\hline & & 15 & Articular competição com plano plurianual. & 0,742 & & & \\
\hline & & 5 & Organizar e implementar o plano anual. & 0,610 & & & \\
\hline & & 2 & $\begin{array}{l}\text { Realizar o plano anual, considerando as } \\
\text { necessidades do atleta/grupo. }\end{array}$ & 0,545 & & & \\
\hline & & 8 & $\begin{array}{l}\text { Avaliar e modificar o planeamento anual, } \\
\text { adaptando-o a imprevistos. }\end{array}$ & 0,488 & & & \\
\hline & & 14 & Articular a competição com o plano anual. & 0,450 & & & \\
\hline \multirow[t]{4}{*}{2} & \multirow{4}{*}{$\begin{array}{l}\text { Competências } \\
\text { de Liderança e } \\
\text { de Formação de } \\
\text { Treinadores }\end{array}$} & 19 & $\begin{array}{l}\text { Coordenar a formação de técnicos da mo- } \\
\text { dalidade. }\end{array}$ & 0,794 & \multirow[t]{4}{*}{0,835} & \multirow[t]{4}{*}{2,646} & \multirow[t]{4}{*}{11,506} \\
\hline & & 17 & $\begin{array}{l}\text { Liderar uma organização, coordenando a } \\
\text { actividade de atletas, treinadores e especia- } \\
\text { listas de ciências do desporto. }\end{array}$ & 0,787 & & & \\
\hline & & 18 & $\begin{array}{l}\text { Orientar a formação de treinadores princi- } \\
\text { piantes. }\end{array}$ & 0,768 & & & \\
\hline & & 16 & $\begin{array}{l}\text { Assumir o papel de treinador principal e co- } \\
\text { ordenar a actividade de outros treinadores. }\end{array}$ & 0,753 & & & \\
\hline \multirow[t]{3}{*}{3} & \multirow{3}{*}{$\begin{array}{l}\text { Competências } \\
\text { de Planeamento } \\
\text { e Orientação da } \\
\text { Competição }\end{array}$} & 13 & $\begin{array}{l}\text { Dirigir um atleta/equipa durante uma com- } \\
\text { petição. }\end{array}$ & 0,738 & \multirow[t]{3}{*}{0,722} & \multirow[t]{3}{*}{1,887} & \multirow[t]{3}{*}{8,203} \\
\hline & & 10 & $\begin{array}{l}\text { Preparar um atleta/equipa para participar } \\
\text { numa competição oficial. }\end{array}$ & 0,716 & & & \\
\hline & & 11 & $\begin{array}{l}\text { Preparar a competição, estabelecendo } \\
\text { objectivos adequados às necessidades do } \\
\text { atleta/equipa. }\end{array}$ & 0,637 & & & \\
\hline \multirow[t]{4}{*}{4} & \multirow{4}{*}{$\begin{array}{l}\text { Competências } \\
\text { Pessoais }\end{array}$} & 21 & Comunicar ideias, problemas e soluções. & 0,820 & \multirow[t]{4}{*}{0,768} & \multirow[t]{4}{*}{1,448} & \multirow[t]{4}{*}{6,295} \\
\hline & & 22 & $\begin{array}{l}\text { Resolver problemas em situações inespera- } \\
\text { das. }\end{array}$ & 0,777 & & & \\
\hline & & 20 & $\begin{array}{l}\text { Responsabilizar-se pela visão do mundo, } \\
\text { preocupando-se com os outros. }\end{array}$ & 0,631 & & & \\
\hline & & 23 & $\begin{array}{l}\text { Aprender autonomamente, adoptando uma } \\
\text { atitude proactiva e reflexiva. }\end{array}$ & 0,596 & & & \\
\hline \multirow[t]{3}{*}{5} & \multirow{3}{*}{$\begin{array}{l}\text { Competências de } \\
\text { Orientação do } \\
\text { Treino }\end{array}$} & 7 & $\begin{array}{l}\text { Avaliar e modificar o plano da sessão de } \\
\text { treino, adaptando-o a imprevistos. }\end{array}$ & 0,642 & \multirow{3}{*}{0,736} & \multirow{3}{*}{1,321} & \multirow{3}{*}{5,742} \\
\hline & & 1 & $\begin{array}{l}\text { Planear a sessão de treino em função das } \\
\text { necessidades do atleta/grupo. }\end{array}$ & 0,616 & & & \\
\hline & & 4 & Organizar e conduzir a sessão de treino. & 0,532 & & & \\
\hline
\end{tabular}


tigação tem concedido escassa atenção às questões relacionadas com o planeamento do treino. Da investigação recente, ressaltam duas dimensões importantes a incorporar neste nível: o delineamento tanto a longo como a curto prazo ${ }^{11}$, tal como indica o programa canadiano de certificação de treinadores $\mathrm{NCCP}^{4}$ que descreve o traçado de planos anuais e plurianuais como uma competência profissional essencial; e a planificação dinâmica, ou seja, a capacidade de prever no planeamento situações inesperadas, sendo importante conceber planos sustentados em atitudes proactivas.

O presente estudo mostra, ainda, que as Competências de Liderança e de Formação de Treinadores, as quais integram as capacidades de coordenação da actividade ao nível das organizações desportivas e de participação na formação de treinadores, foram muito valorizadas pelos treinadores. A gestão é uma competência reconhecida por diferentes projectos de desenvolvimento desportivo e por programas de formação de treinadores, em sede de sistema universitário ${ }^{4,13}$. Estes indicam que os treinadores devem ser capazes de desempenhar funções administrativas e de gestão, por exemplo, relacionadas com a gestão de recursos humanos e a liderança de equipas técnicas. Igualmente, treinadores experts entrevistados por Abraham et al. ${ }^{7}$ descreveram-se como líderes de programas desportivos, confirmando a importância atribuída à competência ao nível da gestão e liderança. Por outro lado, a formação de treinadores, paulatinamente, tem vindo a ser considerada como um objecto científico de estudo, perspectivada como crucial ao incremento da qualidade do processo de treino ${ }^{10,23}$. Importa aqui destacar a importância da formação associada à experiência prática, sobretudo, se supervisionada por treinadores mais experientes, processo que contribui para o alcance da expertise $e^{8,13,16-18}$. Estudos futuros devem debruçar-me mais pormenorizadamente sobre as estratégias de formação no âmbito da formação de treinadores.

$\bigcirc$ terceiro factor emergente associa-se ao contexto da competição, incluindo, não só a orientação dos atletas/equipa, como também o seu planeamento e preparação. Essas competências aparecem, também, valorizadas no âmbito do desenvolvimento de programas de formação universitários ${ }^{4}$. A competição é um contexto crucial no qual se desenrola a actividade desportiva, o que pode explicar o facto de estas competências terem sido, em média, as mais valorizadas pelos treinadores. Tal poderá estar associado ao facto de a performance dos treinadores ser, não raramente, avaliada pelos resultados obtidos, apesar dos objectivos estabelecidos não se restringirem a estes, como destacado pelos treinadores experts do estudo de Abraham et al.? Todavia, um maior investimento na investigação, nomeadamente qualitativa, sobre as competências relacionadas com a orientação e o planeamento da competição poderia fornecer um importante contributo sobre as crenças e valores que sustentam a intervenção dos treinadores, bem como fornecer importantes informações aos responsáveis pela formação destes.

As competências pessoais resultaram no quarto factor, sendo classificadas como possuindo grande importância. Estes aspectos, de índole pessoal e social, são importantes à medida que constituem a base a partir da qual se estabelecem as relações interpessoais e as atitudes e valores do treinador ${ }^{4,13-15}$, com redobrada importância no treinador de jovens.

Finalmente, as competências de orientação do treino emergiram no quinto factor, sendo apontadas pelos treinadores como possuindo grande importância. Estas encontram-se relacionadas com os conhecimentos de cariz didáctico e com a capacidade de aplicação no contexto do treino. A literatura é inequívoca ao afirmar a importância da transmissão de conhecimentos aos atletas, surgindo a instrução como o comportamento mais associado à eficácia na condução do processo de treino ${ }^{22,24,25}$. McCallister et al. ${ }^{26}$, estudando a acção de treinadores de jovens, demonstraram que a falta de competências didácticas pode conduzir a diversas inconsistências entre os valores defendidos e a implementação destes no processo de treino.

De um modo geral, verificou-se que os treinadores com mais experiência atribuem mais importância às competências profissionais, talvez por possuírem uma maior consciência da sua necessidade para o exercício da função, o que reforça a constatação da influência da experiência na competência percebida e efectiva do treinador ${ }^{27}$. Efectivamente, a experiência mostra ser um factor preponderante à aprendizagem do treinador ${ }^{16}$, facto reiterado pelo estudo de Jones et al. ${ }^{8}$, no qual os autores afirmam que a experiência se assume como a principal fonte de desenvolvimento profissional. Assim, evidencia-se a importância de analisar, futuramente, o modo como as experiências pessoais e profissionais influenciam as crenças e os comportamentos do treinador, no âmbito da orientação do processo de treino.

As diferenças encontradas em função da formação académica surgiram entre o grupo de treinadores com habilitações até o $2^{\circ}$ grau com- 
pleto e os grupos com formação superior, sendo que estes últimos, independentemente da formação específica em Educação Física, valorizaram mais as competências profissionais do que os treinadores com habilitações até o $2^{\circ}$ grau completo. Verificouse, portanto, que a formação superior, per se, é um factor qualificador da percepção dos treinadores sobre a valorização das competências profissionais, o que mostra a importância do nível de escolaridade $\mathrm{e}$, consequentemente, da maturidade intelectual no reconhecimento das exigências profissionais. Seria importante o desenvolvimento de investigações de cariz qualitativo, assentes em estudos de caso, de modo a compreender melhor o papel da formação académica nas percepções dos treinadores, bem como perceber o que pode distinguir efectivamente a formação em Educação Física e Desporto relativamente a outras formações superiores.

\section{CONCLUSÃO}

Os resultados obtidos demonstraram a valorização de um alargado leque de competências profissionais relacionadas com o planeamento, a liderança e a formação de outros treinadores, o planeamento e a orientação das competições, aspectos pessoais e a orientação do treino. Assim, sublinha-se o papel multidisciplinar e complexo da actividade de treinador, bem como a importância de uma completa e fundamentada formação profissional.

Apesar de todas as competências terem sido classificadas como, pelo menos, importantes, as competências relacionadas com o contexto da competição foram as mais valorizadas. A experiência profissional demonstrou ser um factor diferenciador da valorização atribuída às competências, sendo que os treinadores mais experientes valorizaram mais as competências relacionadas com o planeamento, a orientação do treino, a liderança e a formação de treinadores do que os com menos experiência. Os treinadores com formação superior, quer em Educação Física e Desporto quer em outras áreas, valorizaram significativamente mais as competências relacionadas com o planeamento, a liderança e a formação de treinadores, a orientação do treino e as competências pessoais.

Sugere-se, então, que a experiência profissional deva ser considerada na formação de treinadores, pelo recurso a treinadores experientes no desempenho de funções de tutoria e de supervisão. Igualmente, a formação académica não pode ser esquecida, porquanto é relevante na qualificação profissional da actividade de treinador.
Finalmente, a investigação futura deve albergar o estudo detalhado das variáveis equacionadas no presente trabalho, recorrendo à análise qualitativa, como entrevistas e observação participativa, de forma a se obter um entendimento mais profundo, mais situado e mais ecológico das competências inerentes à função de treinador de Desporto.

\section{Agradecimentos}

Este artigo integra-se num projecto financiado pela Fundação para a Ciência e a Tecnologia (FCT), Portugal, no âmbito do Programa Operacional Temático Factores de competitividade (COMPETE) do Quadro comunitário de Apoio III e comparticipado pelo Fundo Comunitário Europeu (FEDER) com a seguinte referência: PTDC/DES/67190/2006 e FCOMP-01-0124-FEDER-007177.

\section{REFERÊNCIAS BIBLIOGRÁFICAS}

1. Wright T, Trudel P, Culver D. Learning how to coach: the different learning situations reported by youth ice hockey coaches. Phys Educ Sport Pedagog 2007;12(2):127-44.

2. Jones RL, Wallace M. Another bad day at the training ground: coping with ambiguity in the coaching context. Sport Educ Soc 2005;10(1):119-34.

3. Erickson K, Bruner MW, MacDonald DJ, Côté J. Gaining Insight into Actual and Preferred Sources of Coaching Knowledge. Int J Sport Sci Coach 2008;3(4):527-38.

4. Demers G, Woodburn AJ, Savard C. The Development of an Undergraduate Competency-Based Coach Education Program. Sport Psychol 2006;20(2):162-73.

5. Kirschner P, VanVilsteren P, Hummel H, Wigman M. The design of a study environment for acquiring academic and professional competence. Stud High Educ 1997;22(2):151-71.

6. Westera W. Competences in education: a confusion of tongues. J Curriculum Stud 2001;33(1):75-88.

7. Abraham A, Collins D, Martindale R. The coaching schematic: validation through expert coach consensus. J Sport Sci 2006;24(6):549-64.

8. Jones R, Armour K, Potrac P. Constructing expert knowledge: A case-study of a top-level professional soccer coach. Sport Educ Soc 2003;8(2):213-29.

9. Westera W. On strategies of educational innovation: Between substitution and transformation. High Educ 2004;47(4):501-17.

10. Barnett R. The limits of competence: knowledge, higher education and society. Buckingham: The Society for Research into Higher Education \& Open University Press; 1994.

11. Côté J, Sedgwick WA. Effective Behaviors of Expert Rowing Coaches: A Qualitative Investigation of Canadian Athletes and Coaches. Int Sport J 2003;7(1):62-77. 
12. Côté J, Salmela JH. The organizational tasks of high-performance gymnastics coaches. Sport Psychol 1996;10(3):247-60.

13. Duffy P. Curriculum Model Development - Sport Coaching. In: Petry K, Froberg K, Madella A, editors. Thematic Report Project AEHESIS of the First Year. Cologne: German Sport University; 2006. p. 71-120.

14. Jones RL, Armour KM, Potrac P. Understanding the coaching process: a framework for social analysis. Quest 2002:54(1):35-48.

15. Salmela JH. Great Job Coach! Getting the edge from proven winners. Ottawa: Ed. Potentiun; 1996.

16. Irwin G, Hanton S, Kerwin DG. Reflective practice and the origins of elite coaching knowledge. Reflect Pract 2004;3(5):425-42.

17. Gould D, Giannini J, Krane V, Hodge K. Educational needs of elite U.S. national teams, Pan American, and Olympic Coaches. J Teach Phys Educ 1990;9(4):332-44.

18. Bloom GA, Bush N, Schinke R, Salmela JH. The importance of mentoring in the development of coaches and athletes. Int J Sport Psychol 1998;29(3):267-89.

19. Bloom GA. Characteristics, knowledge, and strategies of expert team sport coaches [Tese de Doutorado $\mathrm{PhD}$ in Education] Ottawa (Ontario): University of Ottawa (Canada); 1997.

20. Burden P. Teacher development. In: Houston WR, editor. Handbook of research on teacher education. New York: Macmillan; 1990. p. 311-27.

21. Tabachnick BG, Fidell LS. Using Multivariate Statistics (5th ed.). Boston: 18 Allyn and Bacon; 2007.
22. Gilbert W, Trudel P. Framing the construction of coaching knowledge in experiential learning theory. SOSOL - Sociol Sport Online 1999;2(1). http://physed. otago.ac.nz/sosol/v2i1/v2i1.htm.

23. Vargas-Tonsing TM. Coaches' preferences for continuing coaching education. International J Sport Sci Coach 2007;2(1):25-35.

24. Mesquita I, Sobrinho A, Rosado A, Pereira F, Milistetd M. A Systematic Observation of Youth Amateur Volleyball Coaches Behaviours. Int J Appl Sport Sci 2008;20(2):37-58.

25. Mesquita I, Rosado A, Januário N, Barroja E. Athlete's retention of a coach's instruction before a judo competition. J Sport Sci Med 2008;7(3):402-7.

26. McCallister SG, Blinde EM, Weiss WM. Teaching values and implementing philosophies: dilemmas of the youth sport coach. Phys Educ 2000;57(1):35-46.

27. Liukkonen J, Laakso L, Telama R. Educational Perspectives of Youth Sport Coaches: Analyses of Observed Coaching Behaviours. Int J Sport Psychol 1996;27(1):439-53.

\section{Endereço para correspondência}

Isabel Mesquita

Rua Dr. Plácido Costa, no 91, 4200.450 - Porto, Portugal

E-mai: imesquita@fade.up.pt 